\title{
Gradation Trajectories of Ideal Initial Printing Colorants in Electrophotography: Discrete Computation
}

\author{
Dmitry A. Tarasov ${ }^{\text {a) }}$, Oleg B. Milder ${ }^{\text {b) }}$ \\ Ural Federal University, Mira str., 19, Ekaterinburg, RUSSIA 620002 \\ a)Corresponding author: datarasov@yandex.ru \\ b)milder@mail.ru
}

\begin{abstract}
Accuracy of the color reproduction during printing is strongly determined by the fine tuning of the gradation curves of the printing systems (tone reproduction curves). The curves are constructed by sequentially changing the tone of the main dyes (most often it is CMYK). However, this approach does not take into account the effect of shading changes when initial colorants overlap. Moreover, the traditional method does not take into account the nonlinearity of the tone increment in the areas of high and low densities. We have proposed an alternative color correction model based on the gradation trajectories as an analogue of gradation curves in the CIE Lab space. The method is devoid of the main drawbacks of the common approach. The authors have previously described the approach, its extension to double overlays of colors (gradation surfaces), as well as its analytical and discrete implications. In this paper, we propose to apply the gradation trajectories as geodesic lines of double overlaps to determine the color of the "true" initial colorants, which are free of nonlinearities in the high density domain. An experimental test was performed using an electrophotographic printer.
\end{abstract}

Keywords: Initial colorant, Gradation trajectories, Gradation surfaces, True color, Digital printing.

\section{INTRODUCTION}

In the color management, the color reproduction adjustment is closely related to the fine tuning of the basic colorants gradations. This approach works in conjunction with various color prediction models embedded in the color management systems and aims to adjust colors, save amount of inks used, and to predict the resulting color in print. Initial colorants in the frame of the standard subtractive color model are represented by three basic colors (CMY) and one additional Black $(\mathrm{K})$. The color formation in such a model is a separate complicated task, although all color prediction models have to rely on those four colorants and their overlapping.

On the other hand, a few is known about the initial colorants in real printing systems. Usually, each manufacturer uses its own recipe of the CMYK inks formation based on the fossil pigments. Hence, each color reproduction system, in fact, uses a unique initial colorants set, especially, considering the non-ideality of the printing system itself. Anyway, each system reproduces quite accurate colors after the procedure of calibration and color tuning with the help of color prediction models used in the color management software is carried out.

There are many different color prediction models applied in color management. The empirical surface models take into account superposition of ink halftones and do not deal with the light propagation and fading within the print. The physically inspired models engage a more detailed analysis of light-print interaction based on the prediction of how the light paths go within a halftone print and what the resulting fade is. The ink spreading models characterize the effective surface of an ink dot after it has been printed at a given nominal surface coverage compared to the effective surface coverage that forms the physical dot gain. The spectral reflection color prediction models deal with spreadbased light propagation-transportation probability. These models study the impact of different factors influencing the range of printable colors (the inks, substrate, illumination conditions, and halftones) and create the printer characterization profiles for the purpose of color management [1]. These models together with the ink-spreading models take into account the physical dot gain and are able to predict the reflectance spectra as a function of ink surface coverage for 2-4 inks (binary and ternary color systems). The models use the multiple tone reproduction (ink

International Conference of Numerical Analysis and Applied Mathematics ICNAAM 2019

AIP Conf. Proc. 2293, 140016-1-140016-4; https://doi.org/10.1063/5.0026742

Published by AIP Publishing. 978-0-7354-4025-8/\$30.00

140016-1 
spreading) curves to characterize the physical dot gain of the ink halftones on the substrate and under all solid inksuperposition conditions [2-4].

The color prediction models have been successfully applied to the color reproduction management [5-9], however, all the proposed approaches require a significant number of measurements, computations and checks. However, since most of them are based on empirical relationships, the accuracy of predicting some shades of the reflection spectrum remains low.

If one looks at the problem of color reproduction from the other side, the use of the "ideal" initial colorants might be an excellent solution providing the basic color stability. An ideal colorant is such a combination of the initial colorants, which ensures minimal fluctuations of color tone in the range from a white substrate to a full dye. In this case, the ideal colorant will be a specific mixture of two initial colorants.

In previous works, the gradation trajectories (GT) as a three-dimensional interpretation of tone reproduction curves (TRC) in CIE Lab space were introduced [10-12]. Thus, any color is uniquely described by a three-component vector in the 3D $L a b$ space. The metric of the space is the color difference $d E$ (or $\Delta E$ ), which is defined at least by the root squared sum of color components differences. Presence of any metric in the sense of the method of measuring distances turns the color space into a metric one.

The GT's arc length is defined as an integral of the $d E$ units. The arc is split into $n$ segments of equal length in order to match them with the same tone increment. We have suggested an empirical description of the GT by polynomials of the $4^{\text {th }}$ degree, as well as the discrete approach to the GT computation on the basis of intrinsic color quantization in the digital printing systems. Further, we expanded the approach onto the binary overlapping of the initial colorants. Thus, the method of gradation surfaces (GS) has been developed [13]. On the basis of the proposed approaches, methods were developed for determining the linearization of various printing systems and determining the gray balance [14]. In the case of printing colorants, their paired double overlays (binaries) correspond to additive primary colors (RGB). Since the RGB and CMYK spaces are both device-dependent, there has not been any simple or general transformation formula that converts them. Using the gradation surface as a gradation trajectory of two colorants, we have suggested the way to develop such a conversion.

If we consider an ideal colorant's GT as a particular geodesic line on the GS of some basic colorants, then we could calculate it using both an analytical and discrete approaches. This work aims to establish a discrete approach for searching the ideal colorants' gradation trajectories based on a color quantization in digital printing systems.

\section{APPROACH AND EXPERIMENT}

Notions of gradation trajectories and gradation surface remain the same. The gradation trajectory is a geometric place of the points in the CIE Lab space, which coordinates correspond to the CIE Lab coordinates of the individual patches of the initial colorants $(\mathrm{C}, \mathrm{M}, \mathrm{Y}, \mathrm{K})$. We assume that the tone part in the layout is denoted as a $t$ parameter in the range from zero (paper) to one (full dye). Therefore, the trajectories might be analytically described by polynomials of $n^{\text {th }}$ degree, since color changes continuously in accordance with continuous increment of $t$. The gradation surface of double colorants overlapping is a surface "stretched" on two gradation trajectories of the initial colorants. Extending the concept of gradation trajectories on the two-dimensional case, we define the gradation surface as a locus of points in the CIE Lab space where coordinates correspond to the CIE Lab coordinates of the individual patches of the binary halftone scale from zero (unprinted substrate) to two (full dye of 2 colorants). By analogy with the gradation trajectory, the gradation surface is also expressed by a polynomial of $n^{\text {th }}$ degree.

Color quantization in digital printing system with the color depth 8 bit per color channel is accounted as a virtual grid of $2^{8}=256$ lines per 1 channel and $2^{16}=65536$ possible halftones of a chosen pair of colorants. The halftone recipes achievable within a given printing system are in the virtual grid nodes. Each reproducible tone has its own recipe $(m$, $n$ ) and a set of the CIE Lab coordinates.

Introducing the color quanta based on the above reasoning, we have to start from the fact that the tone fraction must take values from a particular discrete series. Thus, to account the color quantization in a real print system, the real tone fraction values should be transformed according to the equation $t_{\text {fact }}=u i n t 8\left(t_{\mathrm{des}} \times 255\right) / 255$, where $t_{\mathrm{des}}$ is the desired tone fraction, $t_{\text {fact }}$ is the actually realizable tone fraction, uint 8 is the Matlab function for rounding to an integer in the range $0-255$ (8-bit integer). Thus, each patch recipe component $(m, n)$ is rounded to the 8-bit integer that forms a grid of the color quanta replacing the initial colorant percentage.

We showed that the best trajectory of a binary surface is the geodesic on a gradation surface between $(0,0)$ and $(1,1)$ points. Hence, we will find the gradation trajectory of an ideal colorant assuming that, first, it exists and is 
unique, second, it is a geodesic of two adjacent colorants. The criterion for determining such a trajectory is the minimum color difference $d E_{2000}$ between each successive pair of the color quanta.

For the experiment, we have selected the electrophotographic Konica-Minolta Bizhub Pro C6000L as a printing system with the print resolution $1200 \times 1200$ dpi and the RIP EFI Fiery IC-306. Paper substrate: Moorim Neo Star Matt coated, $140 \mathrm{~g} / \mathrm{m}^{2}$. The test gradation scales were synthesized using the ChartGenerator in the MeasureTool in the ProfileMaker package for the X-Rite il iSis automatic spectrophotometer. The test chart contained 2115 gradation patches that correspond to the 15-quanta tone increment for each colorant forming a binary surface.

Gradation scales with an approximately $5 \%$ increment of the tone in the layout from 0 to $200 \%$ were implemented. Since the tone fraction must take values from the discrete series $[0 ; 0.05 ; 0.1 \ldots 0.95 ; 1]$, the fraction values should be transformed according to the discretization formula (3). The numerator of formula (3) is the expression of color tone in each color quanta. The expression of the color tone in quanta is initially formed in terms of the bit depth of the printing system and is preferable to use. Whichever way of specifying a color is chosen, formula (3) allows establishing an unambiguous relationship between them.

The algorithm used for the sequential quantized recalculation takes place since each of the components of the gradient surface of the color difference increment with respect to the unprinted substrate changes sign at least only once. In other words, the surface of color differences to the substrate color has no local maxima or minima.

For each point of the isoline, the sum of the color differences to the point corresponding to the initial (for instance, unprinted paper) and target points (for instance, full dye Cyan) is computed. Among all these sums corresponding to one $p$, the lowest value has been found. The recipe $(m, n)$ corresponding to this minimal sum at the isoline defines the recipe of the geodesic crossing this isoline. The complete set of points found in this way forms the desired geodesic. This line provides the shortest path from the paper point to the point of complete double overlap. This is the major result of the proposed algorithm.

\section{RESULTS AND DISCUSSION}

The results of the sequential execution of the algorithm for each gradation surface (RGB) and two initial colorants (CMY) depicting the projections of the ideal colorants on the corresponding surfaces are shown in Fig. 3. At the first look, these projections should complement each other in areas where the geodesic of one surface fits its boundary. As follows from Fig. 1, it is not so. This can mean only one thing: the ideal colorant does not lie on any of the surfaces of double overlays but is inside the body of the color gamut of the printing system. In other words, more than two initial colorants that are necessary for its formation.
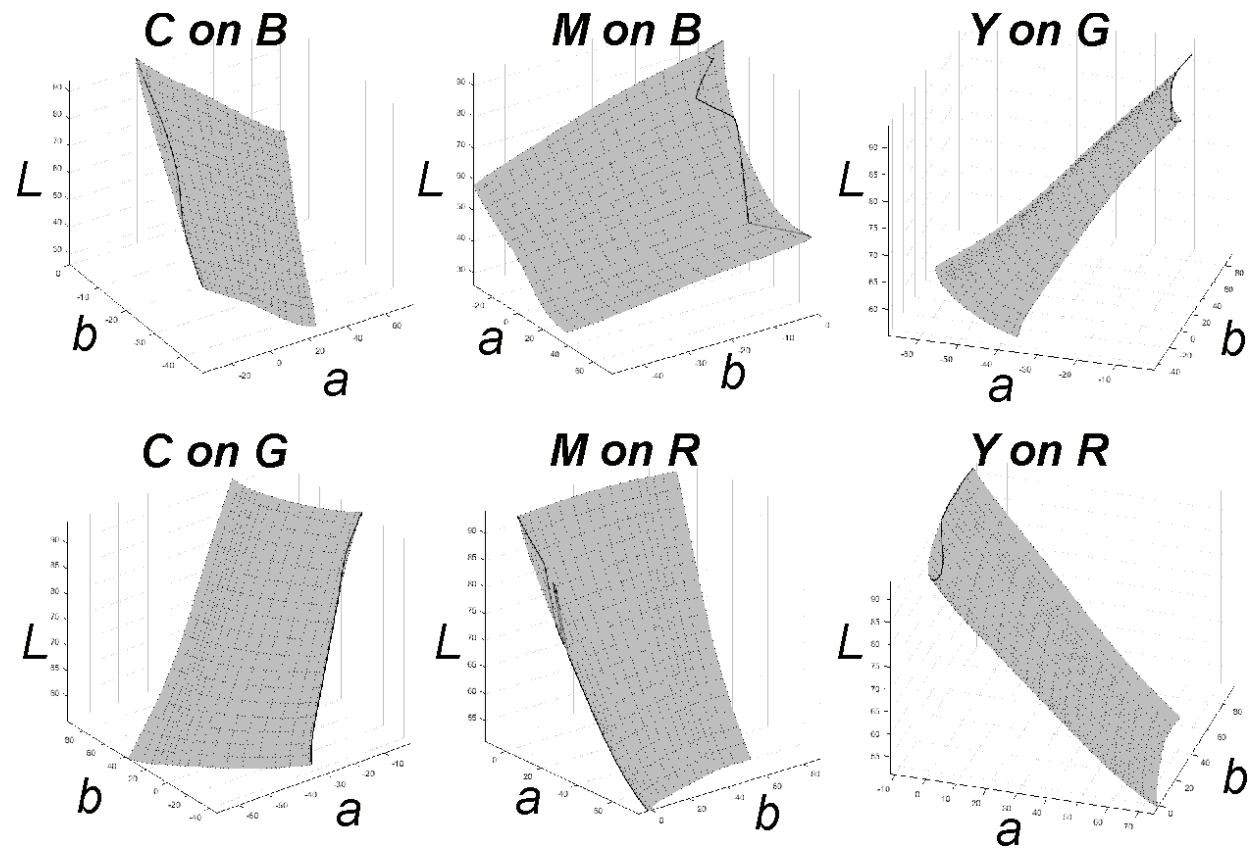

FIGURE 1. Geodesics as the projections of the ideal colorants on the corresponding gradation surfaces 
The projections of the ideal Magenta on the Blue and Red surfaces are of particular interest. The broken character of the projections is related to the fact that the localization area of the ideal Magenta is close to the singularity points of the formula $d E_{2000}$. In the case of electrophotography, it is obvious that modeling of the ideal colorants is at the moment rather speculative. The real implementation of toners with the "ideal" colors for use in printing systems is impossible for reasons that formed the basis of the Yule-Nielson model. When hypothetically creating these ideal colorants, it will be necessary to completely revise the model of subtractive color synthesis.

\section{CONCLUSION}

The work is aimed to create an approach to the ideal colorants definition and calculation. We assume the existing of the relationship between two device-dependent color spaces (CMYK and RGB) accounting the color quantization in print (as for instance, in electrophotography). This makes the systems to be able to precisely express primaries of additive color synthesis $(\mathrm{R}, \mathrm{G}, \mathrm{B})$ as binaries of subtractive color synthesis colorants $(\mathrm{C}, \mathrm{M}, \mathrm{Y})$. For this, the universal CIE Lab color space is used in computations. The binary surface is defined as a gradation surface of two colorants that is "stretched" on their gradation trajectories.

An ideal colorant is such a combination (mixture) of the initial colorants, which ensures the minimal fluctuations of color tone (i.e. color difference) in a range from an unprinted substrate to a full dye. The gradation trajectory of the ideal colorant is the geodesic on the gradation surface of two adjacent initial colorants. The trajectory satisfies the condition of the minimum possible color difference between the color quanta that form it. A discrete interpretation of the ideal colorant computation is proposed in terms of gradation trajectories in the CIE Lab space together with the color quanta as an expression of color quantization.

In the case of electrophotography, the practical implementation of the ideal printed colorant will require a complete revision of the model of subtractive color synthesis, which is hardly in the interests of production. However, this question is extremely interesting from a theoretical point of view. The case of inkjet printing, also, requires particular consideration.

\section{REFERENCES}

1. R. Bala, "Device characterization" in Digital Color Imaging Handbook, edited by G. Sharma (CRC Press, Boca Raton, FL, 2003), pp.269-379.

2. R. Balasubramanian, J Elec. Imag. 8, 156-166 (1999).

3. R.D. Hersch and F. Crété, "Improving the Yule-Nielsen modified spectral Neugebauer model by dot surface coverages depending on the ink superposition conditions" in Proc. SPIE 5667 (2005), pp. 434-445.

4. D.R. Wyble and R.S. Berns, Col.Res.\&App. 25, 4-19 (2000).

5. N.P. Garg, A.K. Singla, and R.D. Hersch, J.Imag.Sci.Tech 52(4), 040908-040908-5 (2008).

6. J.S. Arney, P.G. Engeldrum, and H. Zeng, J.Imag.Sci.Tech 39, 502-508 (1995).

7. S. Livens, "Optimisation of Printer Calibration in the Case of Multi Density Inks" in Conference on Color in Graphics, Imaging, and Vision, CGIV 2002. Final Program and Proceedings (2002), pp. 633-638.

8. L. Chagas, A. Blayo, and P. Giraud "Color Profile: methodology and influence on the performance of ink-jet color reproduction" in IS\&T's NIP20. International Conference on Digital Printing Technologies (2004), pp. 655659.

9. Y-J. Wu Reducing "Ink-jet Ink Consumption with RIP software for POP Display Media. Digital Fabrication and Digital Printing" in NIP30 Technical Program and Proceedings (2014), pp. 108-111.

10. M. Titova, O. Milder, and D. Tarasov "Gradation trajectories: acquisition and analysis" in Proceedings of the Conference «Information: transmission, processing, perception». UrFU (2016) pp. 120-125 (in Russian).

11. O.B. Milder, D.A. Tarasov, and M.Yu Titova "Inkjet Printers Characterization Using 3D Gradation Curves" in CEUR Workshop Proceedings. Vol.1814 (2017) pp. 74-83.

12. O. Milder and D. Tarasov "Ink-jet printer's characterization by 3D gradation trajectories on an equidistant color difference basis". Lecture Notes in Computer Science, vol. 10749 (2018) pp. 40-52.

13. O. Milder and D. Tarasov "Gradation surfaces as a method for multi-color ink-jet printers color specifications management" in Lecture Notes in Computer Science (LNCS), vol. 10749 (2018) pp. 53-61.

14. D.A. Tarasov and O.B. Milder, Comp. Optics 43(1), 132-136 (2019) (In Russian). 